\title{
Variables hidrometereológicas asociadas al cambio climático en Girardot y la Región del Alto Magdalena*
}

\section{Hydrometereological Variables Associated to Climate Change in Girardot and the Region}

\author{
Variables hydrométéorologiques associées au \\ changement climatique à Girardot et la Région
}

\author{
Genaro Penagos-Cruz**
}

Recibido: 2014-03-12 // Aprobado: 2014-05-08 // Disponible en linea: 2014-09-10

Cómo citar este artículo: Penagos Cruz, G. (2014). Variables hidrometereológicas asociadas al cambio climático en Girardot y la Región del Alto Magdalena. Ambiente y Desarrollo, 18(35), 133-147. http:// dx.doi.org/10.11144/Javeriana.AyD18-35.vhac

doi:10.11144/Javeriana.AyD18-35.vhac

\begin{abstract}
Resumen
Hoy la región del Alto Magdalena cuenta con ocho estaciones de monitoreo, administradas principalmente por el Instituto de Hidrología, Meteorología y Estudios Ambientales (Ideam) y la Corporación Autónoma Regional de Cundinamarca (CAR), lo cual evidencia que existe un registro incompleto de la variables climáticas, razón por lo cual es necesario aplicar una metodología para el arreglo estadístico de datos que combine conceptos como la doble masa, el análisis comparativo del comportamiento, la tendencia y el ajuste estacional, los cuales facilitan la consolidación de la materia prima para la construcción de escenarios de cambio climático a partir de la implementación de simuladores regionales del clima que respondan a la necesidad de evaluar la vulnerabilidad de Girardot frente a esta problemática.
\end{abstract}

Palabras clave: Cambio climático; vulnerabilidad; arreglo estadístico; bases de datos

Este artículo hace parte del proyecto de investigación Evaluación de los costos económicos de cambio climático en Girardot y la región, desarrollado por la Universidad Piloto de Colombia, Seccional Alto Magdalena.

** Ingeniero industrial, especialista en Ingeniería de la Producción, Ms.C. en Educación, docente investigador del programa de Ingeniería Civil, Universidad Piloto de Colombia, Seccional Alto Magdalena. Correo electrónico: Genaro.penagos@gmail.com 


\begin{abstract}
Today the Upper Magdalena region has eight monitoring stations, managed mainly by the Hydrology, Meteorology, and Environmental Studies Institute (IDEAM) and the Autonomous Regional Corporation of Cundinamarca (CAR). These show an incomplete record of the weather variables, creating the need to apply a methodology for the statistic arrangement of data. The aim would be to establish a methodology that combines concepts, such as double mass, comparative behavior analysis, seasonal trend and adjustment. These would ease the consolidation of the raw materials for the construction of climactic change scenarios from the implementation of regional weather simulators that answer the need to assess the vulnerability of Girardot against these problems.
\end{abstract}

Keywords: climate change; vulnerability; statistical arrangement; databases

\title{
Résumé
}

Aujourd'hui la région de l'haute Magdalena compte avec huit stations de monitorage, administrés notamment par l'institut d'Hydrologie, Météorologie et études Environnementales (IDEAM) et la Corporation Autonome Régional de Cundinamarca (CAR), en montrant un registre incomplet des variables climatiques; on perçoit le besoin de l'application d'une méthodologie pour l'arrangement statistique de données, on établit une méthodologie qui mélange les concepts, tels que la double masse, l'analyse comparative du comportement, la tendance et le réglage des stations, qui facilitent la consolidation de la matière première pour la construction de scenarios de changement climatique à partir de l'implémentation de simulateurs régionaux de la météo qui puissent répondre au besoin d'évaluer la vulnérabilité de Girardot face à cette problématique.

Mots-clés: Changement climatique; vulnérabilité; arrangement statistique; base de données. 


\section{Introducción}

El incremento de la temperatura en los últimos años ha despertado la inquietud de la comunidad en general. Tal como lo plantea Carlos Costa (2007) en un artículo sobre la adaptación al cambio climático en Colombia: "[...] durante los últimos 50 años la temperatura promedio de la superficie aumentó $0.65^{\circ} \mathrm{C}$. No puede ser coincidencia que, a partir de 1995 , prácticamente todos los años hayan sido los más calientes de la historia”. Además, la identificación y cuantificación de los factores antropogénicos relacionados con la emisión de gases de efecto invernadero es una tarea primordial para fortalecer los programas de mitigación y adaptación de las poblaciones en general.

La Convención Marco sobre Cambio Climático (cmuncc) del año 2007 propuso a los países miembros la creación de departamento de gestión del riesgo, como factor preponderante para disminuir el impacto del aumento de la temperatura en sectores sensibles como la agricultura y la ganadería (Instituto Internacional de Investigación sobre Políticas Alimentarias IFPRI, 2009). La emanación de los gases de efecto invernadero y su influencia en la atmósfera permitieron asociar el cambio climático global a la fluctuación del clima, posibilitando evidenciar la correlación de la variación en la precipitación, la humedad relativa y el brillo solar con el aumento de la temperatura promedio, que para el caso del valle del Magdalena en el año 2030 tendrá un incremento de $2^{\circ} \mathrm{C}$ (Ideam, 2008).

En este panorama de cambios en el clima, resulta de vital importancia realizar análisis de los datos obtenidos por estaciones meteorológicas a nivel regional, debido a que las proyecciones realizadas por otros estudios muestran datos generales, que en algunos casos no sirven como referente para conocer escenarios específicos de una región.

Las estaciones hidrometereológicas son el medio de suministro de información de las diferentes variables evaluadas. En el territorio en estudio existen varias de estas funcionando y son administradas principalmente por el Instituto de Hidrología, Meteorología y Estudios Ambientales (Ideam) y la Corporación Autónoma Regional de Cundinamarca (CAR), estas entidades cuentan con información de más de 20 años, con periodos muertos por fallas en los equipos o irregularidades en la operación y mantenimiento de los equipos que operan sin procesos de control estandarizados. De allí que sea necesario establecer una metodología para la imputación de datos faltantes que permita contar con bases de datos adecuadas para evaluar posibles escenarios climáticos a nivel regional.

La metodología que se va a emplear consiste en realizar el análisis descriptivo y analítico mediante una revisión de las bases de datos, evaluando diferentes procedimientos para la imputación de datos faltantes. Luego se elaborarán cuadros con el fin de facilitar el análisis de la información y la elaboración de gráficas, para analizar mediante medidas de dispersión y promedios el comportamiento de las variables, buscando establecer funciones que describan dicho comportamiento.

\section{Datos}

Se estableció el mayor número de estaciones hidrometeorológicas que cuantificarán las cuatro variables climáticas necesarias para la construcción del simulador regional del clima. De esta manera, en el Ideam se recogieron los datos de las estaciones Santiago Vila, Сасом 4, Natagaima y Argelia, y Escuela Samper Madrid, Esperanza, La Mesa y Mesitas en la CARB (figura 1). Teniendo en cuenta la procedencia de los datos y su periodicidad, estos se analizarán como series de tiempo para realizar los ajustes de cada variable en cada una de las estaciones, teniendo como referente el promedio por periodo (mes) y los parámetros de dispersión, como la varianza y la desviación estándar que existían entre el conjunto de datos; finalmente, se debe evaluar si el conjunto de datos presenta tendencia por periodo para asignar el método más adecuado para su pronosticación. 


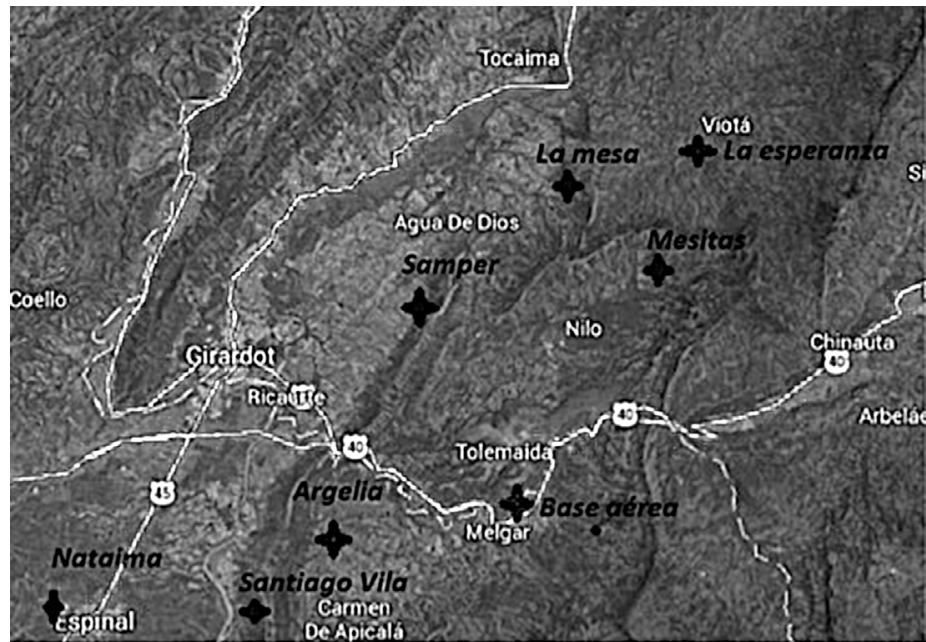

Figura 1. Mapa de ubicación de estaciones meteorológicas en la región de estudio Fuente: https://www.google.com/maps

\section{Aspectos conceptuales}

- Humedad relativa: es el porcentaje de la humedad de saturación, calculada mediante la relación entre la densidad de vapor actual $\left(\mathrm{g} / \mathrm{m}^{3}\right)$ y la densidad de vapor de saturación a la temperatura actual $\left(\mathrm{g} / \mathrm{m}^{3}\right)$, expresado en porcentaje. Esta primera variable se obtiene mediante el empleo de un higrómetro, instrumento utilizado para medir la humedad relativa. Esta representa la cantidad de vapor de agua presente en el aire, y se define como el porcentaje de la cantidad de vapor de agua presente en $1 \mathrm{~m}^{3}$ de aire, a una temperatura determinada:

$$
H \cdot R=\frac{\text { densidad de vapor actual }}{\text { densidad de vapor de saturación }} \times 100 \%
$$

Donde la densidad de vapor (figura 2) está dada por la función D.V $=5,018+0,32321 * T+$ $8,1847^{*} 10^{-3 *} T^{2}+3,1243^{*} 10^{-4 *} T^{3}$ (Cabrera, s. f.).

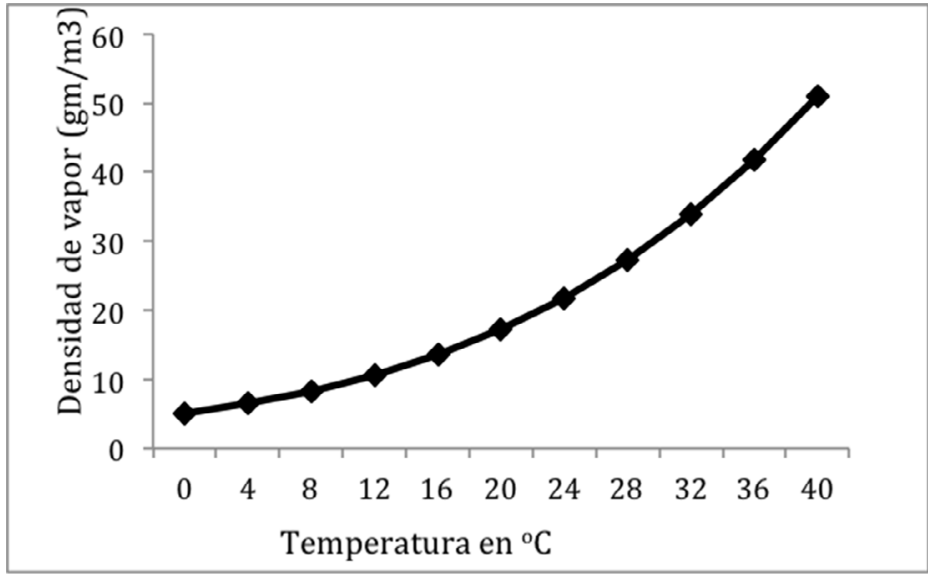

Figura 2. Densidad de vapor

Fuente: elaboración propia 
- Brillo solar: también conocido como heliofanía, representa el tiempo total durante el cual incide la luz solar directa sobre una superficie durante todo el día. La unidad de medida mensual es el resultado de las suma de todos los días del mes.

- Temperatura media: promedio de temperaturas tomadas durante un periodo de tiempo determinado, o el promedio entre la temperatura máxima y mínima (Fraume Restrepo, 2006, p. 426).

- Evaporación: es una separación de componentes por efecto térmico, cambiando de un estado líquido a un estado gaseoso. Puede producirse entre 0 y $100^{\circ}$, a la presión atmosférica; es un índice de un valor significativo, en especial cuando se pretende calcular el balance hídrico.

- Precipitación: caída de partículas sólidas o líquidas de agua sobre una superficie. La cantidad de precipitación, llamada pluviosidad o monto pluviométrico, tiene como unidad milímetros de agua o litros por metro cuadrado. Los instrumentos más empleados para la medición de la lluvia son los pluviómetros y los fluviógrafos.

\section{Imputación de datos faltantes}

Los periodos suministrados por las fuentes primarias (estaciones) presentan grandes irregularidades en la estimación, debido a que difieren en el inicio de su recolección y existen faltantes en los periodos dados en cada una de las variables. Existe una considerable dispersión, lo que representa la sensibilidad del clima a cambios permanentes. Esto implica, de manera significativa, una varianza elevada de los datos y una gran incertidumbre en la predicción de los datos.

Otro aspecto que es preciso evaluar se deriva de la discontinuidad en las bases de datos, las cuales en su mayoría no presentan en forma continua dicho registro, por razones de funcionamiento o mantenimiento. En general, el rango de tiempo no concuerda con las demás estaciones. Además, algunas variables no están presentes en las estaciones, lo que representa una reducción sustancial en el periodo de observación a solo 20 años, con una frecuencia de doce veces por año. Este periodo resulta de establecer un promedio mensual de la variable empleada.

Existen diversas metodologías aplicadas a la imputación de datos faltantes. Una de ellas es la empleada por Urrutia-Palomino (2010), la cual consiste en el empleo de correlaciones parciales, modelos de regresión, ajuste de los datos (método de la doble masa) y verificación de la tendencia a través del test de Kendal. Asimismo, se realiza una apreciación de acuerdo con la cantidad de datos faltantes y se plantea que si el porcentaje es inferior al 20\%, la metodología es aplicable, y si es mayor a este valor se recomiendan otras metodologías como la AMI bivariada.

En un estudio comparativo realizado por Rey (2007) se evalúan diferentes métodos de interpolación para la realización de mapas de precipitación. Se emplea la ecuación:

$$
P x=\frac{\sum_{x=1}^{n} P i r i^{2}}{\sum_{x=1}^{n} r i^{2}}
$$

Esta corresponde a un método de interpolación utilizado para elaborar mapas de precipitación, el cual consiste en tener en cuenta los datos de estaciones cercanas con características similares y el coeficiente de correlación de Pearson de la serie de la estación que se va a completar. Esta metodología se debe aplicar a bases de datos que muestran una buena correlación (Rey, 2007, p. 132). Además, se debe contar con una distribución apropiada, que no es el caso del presente estudio, por la distribución y el funcionamiento.

Infante, Ortega y Cedeńo (2008) proponen un método para reconstrucción de series de tiempo de precipitaciones aplicado a estaciones meteorológicas, cuyo objetivo es los datos faltantes. Este método 
fue desarrollado mediante redes neuronales, partiendo del supuesto de que los datos presentes y los de periodos anteriores pueden utilizarse para predecir precipitaciones hacia adelante (Infante, Ortega y Cedeño, 2008). Se debe tener como restricción la tendencia o comportamiento de los datos en un periodo anual, que en su mayoría guarda un comportamiento con tendencia cíclica.

La Organización Mexicana de Meteorólogos, A. C. (ommac) (2011), en el XX Congreso Mexicano de Meteorología emplea métodos temporales geoestadísticos para estimar datos faltantes en series de tiempo de larga duración para datos de precipitación aplicados a las estaciones climatológicas de la cuenca de un río para un periodo de 25 años. El primer parámetro de relación corresponde a la variación que deben tener los datos entre estaciones cercanas: si la variación está en un 10\%, se aplica el promedio aritmético, y si es mayor se aplica una relación normalizada. Este cálculo es realizado para estaciones que se encuentran alrededor de la estación en estudio y se describen de la siguiente forma:

- El promedio aritmético

$$
D c=\frac{1}{n} \sum_{i=1}^{n} D i
$$

Donde $D c$ es el dato a calcular, $n$ es el número de estaciones cercanas a la estación en estudio y $D i$ corresponde al dato en cada estación.

- Relación normalizada

$$
D p=\frac{1}{n} \sum_{i=1}^{n} \frac{N x}{N i} D i
$$

Donde $N x$ es el dato promedio mensual de la estación en estudio, $N i$ es el dato promedio mensual de las estaciones cercanas que se relacionan y $D i$ es el dato mensual observado en la estación y en la misma fecha del dato faltante por calcular.

Estos métodos resultan muy eficientes y prácticos cuando se cuenta con un número representativo de estaciones ubicadas estratégicamente y equidistantes que ofrecen características y comportamientos muy similares de los datos obtenidos en cada variable en estudio (Campos, 1983).

Los comportamientos que se evalúan, de acuerdo con la periodicidad de la variable, es posible describirlos mediante la utilización de metodologías o técnicas de tipo estadístico. Entre ellas se encuentran:

- El método de la doble masa: permite establecer la homogeneidad de los datos mediante la comparación de periodos que correspondan de acuerdo con la estacionalidad o el comportamiento cíclico.

- El test de Mann-Kendall: método no paramétrico para identificar la tendencia.

- Modelo de regresión lineal: permite identificar la tendencia, predecir mediante el empleo de un modelo teórico y —en caso de no existir una buena relación — obtener una buena aproximación.

- El método de las isoyetas: es utilizado en hidrología para determinar la precipitación media de una cuenca. Consiste en trazar líneas de igual precipitación, denominadas isoyetas, empleando los datos reportados por las estaciones meteorológicas. Para asignar el valor de la precipitación entre dos isoyetas, estas se promedian. Este método ofrece gran confiabilidad cuando las diferencias de altitud son pequeñas y existe una distribución de estaciones en el área de estudio; de lo contrario, aun cuando es de fácil operación, es el menos recomendado. 


\section{Metodología}

Evaluando las diferentes metodologías, diseñadas y aplicadas a un territorio con cobertura más amplia, y teniendo en cuenta el comportamiento y estado de las bases de datos, es posible establecer una combinación de algunas de estas metodologías: la comparación de periodos correspondientes, el modelo de regresión lineal y los ajustes estacionales, que guardan un comportamiento cíclico a pesar de la gran variación de los datos, lo cual es característico en las series de tiempo.

Se parte de que la relación periodo vs. variable meteorológica se puede representar mediante la ecuación general de la recta, para lo cual se empleará el método de mínimos cuadrados, a fin de calcular los coeficientes de la función:

$$
y=a x+b
$$

Donde:

$$
\begin{array}{r}
\frac{\partial F}{\partial b}=\sum \frac{\partial}{\partial b}(\mathrm{ax}+\mathrm{b}-\mathrm{y})^{2}=\sum 2(\mathrm{ax}+\mathrm{b}-\mathrm{y}) \\
\frac{\partial F}{\partial \mathrm{a}}=\sum \frac{\partial}{\partial \mathrm{a}}(\mathrm{ax}+\mathrm{b}-\mathrm{y})^{2}=\sum 2 \mathrm{x}(\mathrm{ax}+\mathrm{b}-\mathrm{y})
\end{array}
$$

Simplificando, se obtiene:

$$
\begin{gathered}
\sum(\mathrm{ax}+\mathrm{b}-\mathrm{y})=0 \text { y } \sum x(\mathrm{ax}+\mathrm{b}-\mathrm{y})=0 \\
\sum \mathrm{y}=\mathrm{an}+\mathrm{b} \sum \mathrm{x} \text { y } \sum \mathrm{xy}=\mathrm{a} \sum \mathrm{x}+\mathrm{b} \sum x^{2}
\end{gathered}
$$

Ecuaciones que permiten calcular el valor de los coeficientes de la función lineal (Spiegel, Schiller $\&$ Srinivasan, 2010, p. 276). Teniendo en cuenta la variabilidad de los datos, es necesario aplicar los conceptos de varianza y desviación estándar, calculados a partir de la siguiente expresión (Nieves \& Dominig, 2010, p. 210):

$$
\sigma^{2}=\frac{\sum_{i=1}^{n}\left(x_{i}-\mu\right)^{2}}{n}
$$

El análisis de tendencia mostrado mediante la ecuación de la recta, debido a las fluctuaciones mostradas en el periodo evaluado, requiere ser ajustado teniendo en cuenta los patrones y la estacionalidad que estos presentan. El comportamiento no es totalmente lineal, lo que implica que un ajuste estacional aproxima más al comportamiento de la variable y su característica fluctuante. Esto se realiza a través de un número índice (índice estacional), el cual se expresa en porcentaje y refleja los cambios a través del tiempo (Hanke \& Reitsch, 1996, p. 339).

Este número índice se calcula en función del promedio mensual de los periodos de estudio:

$$
I E_{i}=\frac{x_{i}}{\frac{\sum_{i=1}^{n} x_{i}}{n}} \cdot 100
$$

Este índice responde al comportamiento del periodo analizado en función del comportamiento de los datos suministrados durante el periodo de estudio. El resultado final aplicado al dato faltante resulta de multiplicar la tendencia por el índice correspondiente del periodo.

$$
\text { Dato calculado }=(\mathrm{a} x+b) * I E i
$$




\section{Completando los datos}

Teniendo en cuenta lo anterior, las variables que se emplearán son aquellas que cuentan con un valor menor al $20 \%$ de faltantes para realizar el análisis descriptivo y analítico, el cual consiste en la descripción y el establecimiento de correlaciones. Estas variables son: humedad relativa (tabla 1), precipitación, temperatura y brillo solar. Inicialmente, se evalúa la "humedad relativa" en las estaciones de la CAR, para mostrar la metodología empleada para completar los datos.

Tabla 1. Variable humedad relativa

C A R - CORPORACIÓN AUTÓNOMA REGIONAL DE CUNDINAMARCA SICLICA - Sistema de Información Climatológica e Hidrológica

VALORES MEDIOS MENSUALES DE HUMEDAD RELATIVA (\%)

\begin{tabular}{|c|c|c|c|c|c|c|c|}
\hline \multirow[b]{2}{*}{ Latitud } & \multirow[b]{2}{*}{$0419 \mathrm{~N} \quad \mathrm{X}=\mathrm{N}=974200$} & \multirow[b]{2}{*}{ Departamento } & \multirow[b]{2}{*}{ CUNDINAMARCA } & \multicolumn{3}{|c|}{ ESTACIÓN : 2120644 ARGELIA } & \multirow[b]{2}{*}{$\mathrm{CP}$} \\
\hline & & & & Corriente & R. BOGOTÁ & Categoria & \\
\hline Longitud & $7441 \mathrm{~W} Y=E=925400$ & Municipio & RICAURTE & Cuenca & R. BOGOTA & Fecha Instalación & 12/01/1991 \\
\hline Elevación & 320 m.s.n.m & Oficina Provincial & 3 ALTO MAGDALENA & & & Fecha Suspensión & \\
\hline
\end{tabular}

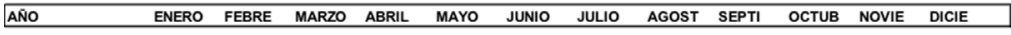

\begin{tabular}{|lllllllllllll|}
\hline 1992 & 62 & 58 & 57 & 71 & 79 & 64 & 58 & 53 & 61 & 64 & 71 & 67 \\
1993 & 73 & 75 & 75 & 79 & 79 & 65 & 59 & 64 & & & & \\
1994 & 73 & 74 & & & 79 & & & 57 & & & & 71 \\
1995 & & & & 79 & 75 & 77 & 69 & 68 & 65 & 73 & 74 & 65 \\
1996 & 70 & 71 & 68 & 75 & 74 & 72 & 64 & 54 & & 71 & 70 & 48 \\
1997 & 58 & 59 & & 71 & 67 & 69 & 54 & 49 & 55 & 64 & 63 & 71 \\
1998 & 59 & 67 & 68 & 76 & 77 & & & & 66 & 68 & 73 & 62 \\
1999 & 70 & 72 & 67 & 68 & 69 & 69 & 54 & 46 & 57 & 64 & 61 & 64 \\
2000 & 53 & 64 & 60 & 65 & 65 & 64 & 52 & 46 & 57 & 57 & 65 & 61 \\
2001 & 51 & 58 & 66 & 60 & 59 & 54 & 50 & 47 & 53 & 60 & & 71 \\
2002 & & 47 & 52 & 63 & 53 & 55 & 48 & 38 & 46 & 52 & 61 & 57 \\
2003 & 54 & 58 & 64 & 71 & 60 & 66 & 55 & 41 & 42 & 69 & 74 & 59 \\
2004 & 50 & 51 & 49 & 62 & 65 & 51 & 50 & 42 & 62 & 71 & 73 & 69 \\
2005 & 71 & 69 & 68 & 69 & 71 & 51 & 46 & 43 & 41 & 60 & 65 & 58 \\
2006 & 53 & 50 & 68 & 71 & 52 & 44 & 36 & 34 & 44 & 54 & 59 & 59 \\
2007 & 49 & 31 & 50 & 63 & & & 71 & 70 & 64 & 71 & 76 & 70 \\
2008 & 70 & 72 & 66 & 79 & 80 & 79 & 70 & 73 & 73 & 78 & 81 & 73 \\
2009 & 76 & 74 & 80 & 77 & 76 & 72 & 63 & 63 & 60 & 64 & 74 & 70 \\
2010 & 64 & 64 & 63 & 75 & 77 & 78 & 76 & 64 & 67 & 67 & 70 & 68 \\
2011 & 60 & 65 & 64 & & & & & & & & & \\
\hline
\end{tabular}

Fuente: bases de datos suministradas por la CAR

Para evaluar los datos faltantes en las bases suministradas, estos se agrupan por periodo (mes), teniendo en cuenta que existe una relación visible del comportamiento en cada uno de ellos, tal como se muestra en las figuras 3, 4 y 5, donde se indica la correlación de los datos para la variable humedad relativa de la estación Argelia-CAR para el periodo de estudio.

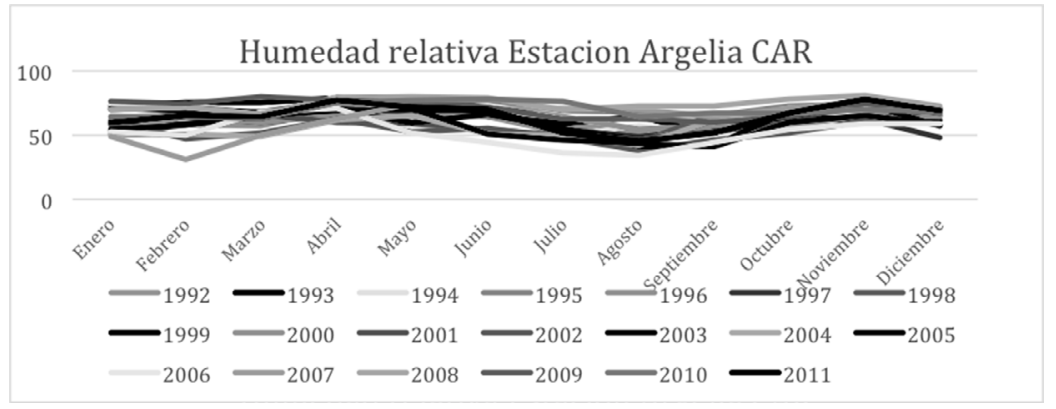

Figura 3. Comportamiento variable humedad relativa, Estación Argelia-cAR Fuente: elaboración propia 
$\mathrm{Al}$ analizar el comportamiento, individualmente para cada periodo (mes), se puede evaluar el comportamiento creciente, el cual tiene una gran correlación con el análisis de tendencia. Sin embargo, es necesario plantear un ajuste, debido a la variación común en este tipo de variables.

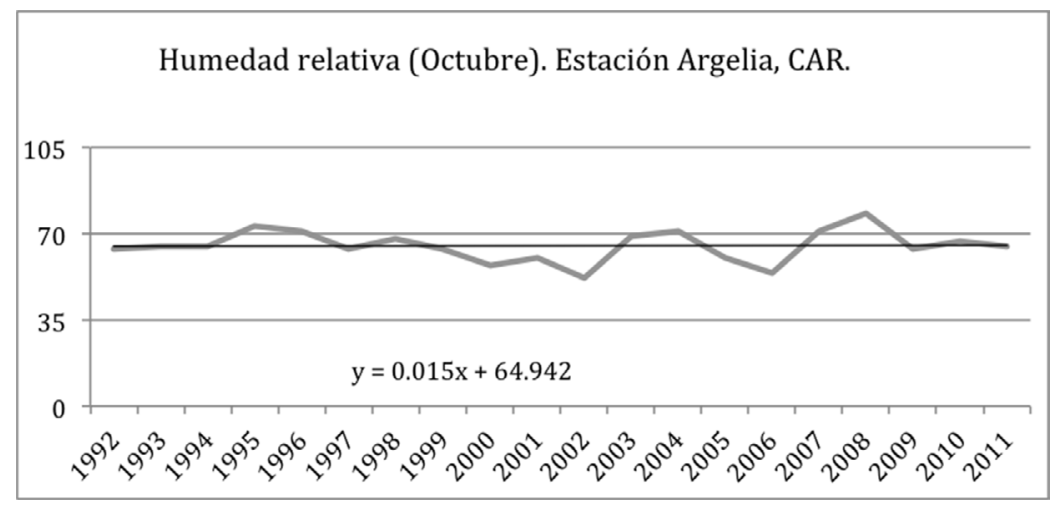

Figura 4. Datos del mes de octubre de la variable humedad relativa. Estación Argelia-CAR

Fuente: elaboración propia

Lo anterior permite conocer la tendencia y comparar por regresión lineal los datos ajustados con respecto al promedio de cada periodo evaluado (mes) (Guarín, 2002), y de esta forma calcular los datos faltantes en la base de datos, teniendo en cuenta que no es posible simular con valores cero (0) en algunos periodos, cuando se realizan simulaciones, objetivo principal del presente estudio.

Emplear la regresión cuando existe tendencia cíclica requiere realizar un ajuste mediante índices estacionales, estos permiten ajustar los resultados de la regresión con el comportamiento de la variable en cada periodo, en forma individualizada.

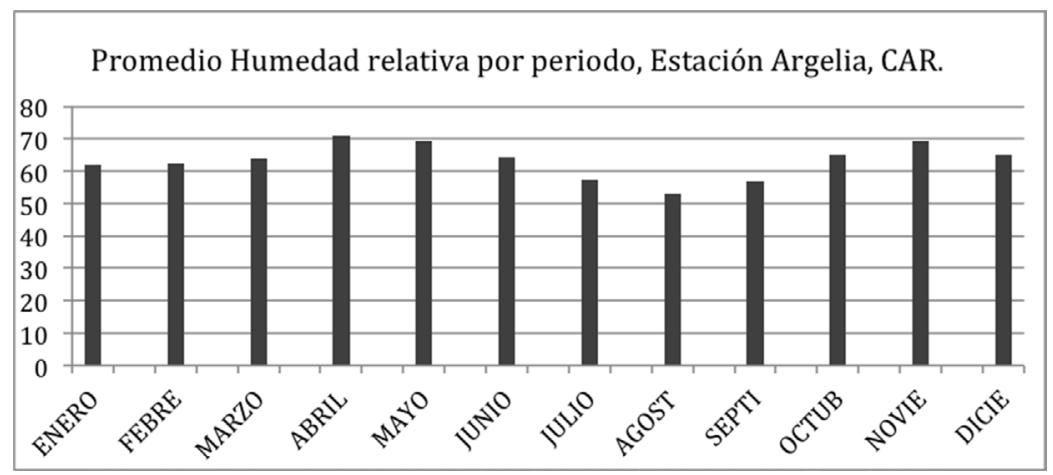

Figura 5. Datos promedio por mes de la variable humedad relativa. Estación Argelia-CAR

Fuente: elaboración propia 
Este proceso de análisis nos lleva a la tabla de datos que se empleará finalmente, En este caso la variable humedad relativa, estación Argelia-CAR (tabla 2).

Tabla 2. Base de datos variable humedad relativa, estación Argelia-CAR

\begin{tabular}{|c|c|c|c|c|c|c|c|c|c|c|c|c|}
\hline \multirow{3}{*}{$\begin{array}{l}\text { Latitud } \\
\text { Longitud } \\
\text { Elevación }\end{array}$} & \multirow{2}{*}{\multicolumn{2}{|c|}{$0419 \mathrm{~N} \quad \mathrm{X}=\mathrm{N}=974200$}} & & \multirow{2}{*}{\multicolumn{2}{|c|}{ CUNDINAMARCA }} & \multicolumn{6}{|c|}{ ESTACIÓN : 2120644 ARGELLA } \\
\hline & & & & & & & Corriente & \multicolumn{2}{|c|}{ R. BOGOTÁ } & \multicolumn{2}{|l|}{ Categoría } & \multirow{2}{*}{$\begin{array}{l}\mathrm{CP} \\
\end{array}$} \\
\hline & $7441 \mathrm{~W} Y=E=925400$ & & Oficina P & Provincial & 3 ALTOMA & GDALENA & & & & Fecha Susp & ension & \\
\hline AÑO & ENERO & FEBRE & MARZO & ABRIL & MAYO & JUNIO & JULIO & AGOST & SEPTI & OCTUB & NOVIE & DICIE \\
\hline 1992 & 62 & 58 & 57 & 71 & 79 & 64 & 58 & 53 & 61 & 64 & 71 & 67 \\
\hline 1993 & 73 & 75 & 75 & 79 & 79 & 65 & 59 & 64 & 57 & 65 & 67 & 63 \\
\hline 1994 & 73 & 74 & 64 & 72 & 79 & 65 & 56 & 57 & 57 & 65 & 68 & 63 \\
\hline 1995 & 64 & 65 & 64 & 79 & 75 & 77 & 69 & 68 & 65 & 73 & 74 & 71 \\
\hline 1996 & 70 & 71 & 68 & 75 & 74 & 72 & 64 & 54 & 57 & 71 & 70 & 65 \\
\hline 1997 & 58 & 59 & 64 & 71 & 67 & 69 & 54 & 49 & 55 & 64 & 63 & 48 \\
\hline 1998 & 59 & 67 & 68 & 76 & 77 & 65 & 57 & 53 & 66 & 68 & 73 & 71 \\
\hline 1999 & 70 & 72 & 67 & 68 & 69 & 69 & 54 & 46 & 57 & 64 & 61 & 62 \\
\hline 2000 & 53 & 64 & 60 & 65 & 65 & 64 & 52 & 46 & 57 & 57 & 65 & 64 \\
\hline 2001 & 51 & 58 & 66 & 60 & 59 & 54 & 50 & 47 & 53 & 60 & 69 & 65 \\
\hline 2002 & 62 & 47 & 52 & 63 & 53 & 55 & 48 & 38 & 46 & 52 & 61 & 71 \\
\hline 2003 & 54 & 58 & 64 & 71 & 60 & 66 & 55 & 41 & 42 & 69 & 74 & 57 \\
\hline 2004 & 50 & 51 & 49 & 62 & 65 & 51 & 50 & 42 & 62 & 71 & 73 & 69 \\
\hline 2005 & 71 & 69 & 68 & 69 & 71 & 51 & 46 & 43 & 41 & 60 & 65 & 58 \\
\hline 2006 & 53 & 50 & 68 & 71 & 52 & 44 & 36 & 34 & 44 & 54 & 59 & 59 \\
\hline 2007 & 49 & 31 & 50 & 63 & 67 & 64 & 71 & 70 & 64 & 71 & 76 & 70 \\
\hline 2008 & 70 & 72 & 66 & 79 & 80 & 79 & 70 & 73 & 73 & 78 & 81 & 73 \\
\hline 2009 & 76 & 74 & 80 & 77 & 76 & 72 & 63 & 63 & 60 & 64 & 74 & 70 \\
\hline 2010 & 64 & 64 & 63 & 75 & 77 & 78 & 76 & 64 & 67 & 67 & 70 & 68 \\
\hline 2011 & 60 & 65 & 64 & 69 & 65 & 63 & 59 & 54 & 57 & 65 & 71 & 67 \\
\hline PROMEDIO & 62 & 62,053 & 63,824 & 70,778 & 69,833 & 64,375 & 57,353 & 52,889 & 57,059 & 65,118 & 69,375 & 65,188 \\
\hline RANGO & 27 & 44 & 31 & 19 & 28 & 35 & 40 & 39 & 32 & 26 & 22 & 25 \\
\hline VARIANZA & 78 & 123 & 64,028 & 36,84 & 78,472 & 105,61 & 102,7 & 131,88 & 81,82 & 45,751 & 37,484 & 43,652 \\
\hline DESVIACIÓN & 8,8318 & 11,09 & 8,0017 & 6,0696 & 8,8585 & 10,277 & 10,134 & 11,484 & 9,0454 & 6,7639 & 6,1224 & 6,607 \\
\hline IE & 0,98 & 0,98 & 1,0079 & 1,1178 & 1,1029 & 1,0167 & 0,9058 & 0,8353 & 0,9011 & 1,0284 & 1,0956 & 1,0295 \\
\hline
\end{tabular}

Fuente: elaboración propia

Tabla 3. Base de datos variable humedad relativa, estación Escuela Samper, Madrid

\begin{tabular}{|c|c|c|c|c|c|}
\hline \multirow{4}{*}{$\begin{array}{l}\text { Latitud } \\
\text { Longitud } \\
\text { Elevación }\end{array}$} & \multirow{3}{*}{$\begin{array}{l}431 \mathrm{~N} \\
435 \mathrm{~W}\end{array}$} & \multirow{3}{*}{$\begin{array}{l}X=N=988200 \\
Y=E=948800\end{array}$} & & \multicolumn{2}{|l|}{ ESTACIÓN : 2120641 ESC SAMPER MADRID } \\
\hline & & & Departamento CUNDINAMARCA & Comiente. CALANDAMMA Categoría & \\
\hline & & & Municipio & Cuencaz. CALANDAIMPFecha Instalación & \#\#\# \\
\hline & m.s.n.m & & Oficina Provincial 3 TEQUENDAMA & Fecha Suspensión & \\
\hline
\end{tabular}

\begin{tabular}{|c|c|c|c|c|c|c|c|c|c|c|c|c|c|}
\hline ANO & & ENERO & FEBRE & MARZO & ABRIL & MAYO & JUNIO & JULIO & AGOST & SEPTI & OCTUB & NOVIE & DICIE \\
\hline 1991 & & 78 & 76 & 80 & 82 & 85 & 83 & 76 & 80 & 84 & 83 & 85 & 88 \\
\hline 1992 & & 87 & 86 & 82 & 85 & 86 & 79 & 76 & 75 & 79 & 80 & 83 & 86 \\
\hline 1993 & & 86 & 81 & 83 & 84 & 86 & 81 & 78 & 74 & 79 & 80 & 87 & 86 \\
\hline 1994 & & 79 & 83 & 83 & 86 & 83 & 82 & 79 & 74 & 76 & 83 & 86 & 83 \\
\hline 1995 & & 76 & 72 & 85 & 85 & 80 & 85 & 83 & 84 & 83 & 85 & 83 & 83 \\
\hline 1996 & & 84 & 84 & 84 & 82 & 85 & 84 & 78 & 74 & 78 & 83 & 83 & 84 \\
\hline 1997 & & 81 & 82 & 75 & 83 & 82 & 83 & 74 & 66 & 71 & 77 & 82 & 82 \\
\hline 1998 & & 83 & 82 & 81 & 84 & 82 & 78 & 75 & 77 & 81 & 74 & 73 & 85 \\
\hline 1999 & & 83 & 84 & 70 & 79 & 76 & 77 & 79 & 80 & 84 & 87 & 89 & 95 \\
\hline 2000 & & 83 & 87 & 88 & 90 & 83 & 82 & 86 & 78 & 83 & 84 & 84 & 86 \\
\hline 2001 & & 83 & 84 & 87 & 86 & 85 & 83 & 77 & 72 & 80 & 62 & 58 & 96 \\
\hline 2002 & & 84 & 81 & 69 & 66 & 80 & 80 & 71 & 75 & 74 & 79 & 76 & 81 \\
\hline 2003 & & 65 & 65 & 64 & 59 & 64 & 67 & 81 & 82 & 82 & 82 & 83 & 87 \\
\hline 2004 & & 84 & 80 & 80 & 84 & 85 & 82 & 80 & 81 & 81 & 83 & 85 & 98 \\
\hline 2005 & & 84 & 81 & 80 & 84 & 85 & 82 & 81 & 82 & 82 & 84 & 85 & 98 \\
\hline 2006 & & 83 & 82 & 81 & 83 & 83 & 82 & 82 & 83 & 82 & 83 & 87 & 87 \\
\hline 2007 & & 94 & 79 & 84 & 86 & 83 & 82 & 82 & 84 & 82 & 83 & 75 & 90 \\
\hline 2008 & & 88 & 75 & 78 & 76 & 89 & 90 & 87 & 91 & 89 & 92 & 94 & 83 \\
\hline 2009 & & 91 & 88 & 93 & 89 & 91 & 82 & 83 & 95 & 82 & 87 & 92 & 88 \\
\hline 2010 & & 79 & 88 & 87 & 91 & 86 & 87 & 87 & 83 & 83 & 85 & 86 & 87 \\
\hline 2011 & & 80 & 83 & 83 & 91 & 83 & 82 & 84 & 87 & 83 & 84 & 83 & 88 \\
\hline & PROMEDIO & 82,619 & 81,095 & 80,81 & 82,619 & 82,952 & 81,571 & 79,952 & 79,857 & 80,857 & 81,905 & 82,81 & 87,667 \\
\hline & VARIANZA & 32,331 & 28,562 & 43,488 & 56,236 & 27,474 & 18,15 & 17,664 & 42,122 & 14,027 & 32,943 & 54,249 & 24,508 \\
\hline & DESCIACIÓN & 5,686 & 5,3444 & 6,5945 & 7,4991 & 5,2416 & 4,2602 & 4,2029 & 6,4902 & 3,7453 & 5,7396 & 7,3654 & 4,9505 \\
\hline & & 1,0068 & 0,9882 & 0,9848 & 1,0068 & 1,0109 & 0,9941 & 0,9743 & 0,9732 & 0,9853 & 0,9981 & 1,0091 & 1,0683 \\
\hline
\end{tabular}

Fuente: elaboración propia 
Tabla 4. Base de datos variable humedad relativa, estación La Esperanza

\begin{tabular}{|c|c|c|c|c|c|c|c|}
\hline \multirow[b]{2}{*}{ Latitud } & \multirow[b]{2}{*}{$0442 \mathrm{~N} \quad X=\mathrm{N}=1012750$} & \multirow[b]{2}{*}{ Departamento } & \multirow[b]{2}{*}{ CUNDINAMARCA } & \multicolumn{4}{|c|}{ ESTACIÓN : 2120647 ESPERANZA LA } \\
\hline & & & & Corriente & R. APULO & Categoría & $C P$ \\
\hline Longitud & $7420 W \quad Y=E=961000$ & Municipio & LAMESA & Cuenca & R. APULO & Fecha Instalación & 12/01/1992 \\
\hline Elevación & $1240 \mathrm{~m}$.s.n.m & Oficina Provincial & 13 TEQUENDAMA & & & Fecha Suspensión & \\
\hline
\end{tabular}

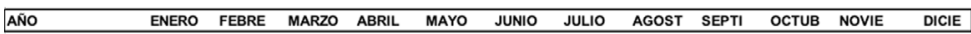

\begin{tabular}{|c|c|c|c|c|c|c|c|c|c|c|c|c|}
\hline 1992 & 72 & 76 & 80 & 79 & 78 & 77 & 71 & 68 & 74 & 79 & 82 & 78 \\
\hline 1993 & 68 & 76 & 80 & 79 & 78 & 77 & 72 & 68 & 73 & 75 & 82 & 76 \\
\hline 1994 & 75 & 75 & 82 & 78 & 79 & 76 & 72 & 68 & 74 & 79 & 83 & 77 \\
\hline 1995 & 68 & 67 & 82 & 83 & 82 & 82 & 76 & 78 & 75 & 82 & 85 & 82 \\
\hline 1996 & 80 & 81 & 84 & 83 & 81 & 78 & 74 & 80 & 79 & 87 & 90 & 83 \\
\hline 1997 & 84 & 87 & 79 & 82 & 78 & 83 & 77 & 67 & 74 & 79 & 87 & 7 \\
\hline 1998 & 68 & 73 & 76 & 78 & 77 & 72 & 70 & 69 & 73 & 79 & 79 & 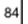 \\
\hline 1999 & 73 & 89 & 83 & 80 & 74 & 79 & 68 & 64 & 77 & 85 & 85 & 8 \\
\hline 2000 & 75 & 74 & 79 & 77 & 81 & 77 & 71 & 67 & 74 & 69 & 73 & 77 \\
\hline 2001 & 70 & 77 & 83 & 74 & 79 & 72 & 65 & 55 & 67 & 73 & 81 & 91 \\
\hline 2002 & 74 & 67 & 71 & 83 & 74 & 72 & 70 & 62 & 64 & 69 & 73 & 79 \\
\hline 2003 & 64 & 72 & 72 & 78 & 81 & 78 & 68 & 70 & 76 & 84 & 87 & 86 \\
\hline 2004 & 79 & 78 & 74 & 87 & 84 & 73 & 76 & 70 & 77 & 92 & 81 & 74 \\
\hline 2005 & 69 & 73 & 79 & 82 & 81 & 78 & 73 & 70 & 73 & 79 & 84 & \\
\hline 2006 & 74 & 74 & 80 & 79 & 76 & 76 & 64 & 58 & 73 & 79 & 84 & 83 \\
\hline 2007 & 67 & 53 & 73 & 86 & 81 & 78 & 74 & 79 & 73 & 83 & 86 & 90 \\
\hline 2008 & 83 & 81 & 86 & 84 & 89 & 86 & 80 & 79 & 79 & 83 & 91 & 85 \\
\hline 2009 & 86 & 84 & 85 & 82 & 81 & 78 & 74 & 75 & 66 & 68 & 83 & 76 \\
\hline 2010 & 69 & 77 & 73 & 81 & 80 & 79 & 81 & 75 & 78 & 82 & 87 & 85 \\
\hline 2011 & 79 & 82 & 83 & 83 & 82 & 78 & 74 & 72 & 73 & 79 & 85 & 84 \\
\hline
\end{tabular}

\begin{tabular}{|crrrrrrrrrrrr|}
\hline PROMEDIO & 73,947 & 75,789 & 79,158 & 81 & 79,895 & 77,474 & 72,579 & 69,789 & 73,579 & 79,263 & 83,474 & 81,421 \\
VARIANZA & 38,787 & 62,061 & 20,87 & 10 & 11,463 & 12,881 & 19,296 & 46,061 & 16,033 & 37,668 & 21,302 & 26,349 \\
DESVIACIÒN & 6,2279 & 7,8779 & 4,5683 & 3,1623 & 3,3856 & 3,589 & 4,3928 & 6,7868 & 4,0042 & 6,1374 & 4,6154 & 5,1331 \\
IE & 0,9569 & 0,9807 & 1,0243 & 1,0481 & 1,0338 & 1,0025 & 0,9392 & 0,9031 & 0,9521 & 1,0257 & 1,0801 & 1,0536 \\
\hline
\end{tabular}

Fuente: elaboración propia

Tabla 5. Base de datos variable humedad relativa, estación La Mesa

\begin{tabular}{|c|c|c|c|c|c|c|c|c|c|c|c|c|c|}
\hline \multirow{4}{*}{$\begin{array}{l}\text { Latitud } \\
\text { Longitud } \\
\text { Elevación }\end{array}$} & \multirow{4}{*}{\multicolumn{3}{|c|}{$\begin{array}{l}0438 \mathrm{~N} \quad X=\mathrm{N}=1003360 \\
7426 \mathrm{~W} \quad Y=E=957190 \\
1300 \text { m.s.n.m }\end{array}$}} & \multirow{4}{*}{\multicolumn{2}{|c|}{$\begin{array}{l}\text { Departamento } \\
\text { Municipio } \\
\text { Oficina Provincial }\end{array}$}} & \multirow{3}{*}{\multicolumn{2}{|c|}{$\begin{array}{l}\text { CUNDINAMARCA } \\
\text { LAMESA }\end{array}$}} & \multicolumn{6}{|c|}{ ESTACIÓN : 2120639 MESA LA } \\
\hline & & & & & & & & Corriente & R. APULO & & Categoría & & $\mathrm{CP}$ \\
\hline & & & & & & & & Cuenca & R. APULO & & Fecha Insta & lación & 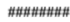 \\
\hline & & & & & & 13 TEQUEI & DAMA & & & & Fecha Susp & ensión & \\
\hline AÑo & & ENERO & FEBRE & MARZO & ABRIL & MAYO & JUNIO & JULIO & AGOST & SEPTI & OCTUB & NOVIE & DICIE \\
\hline 1989 & & 75 & 79 & 82 & 77 & 76 & 73 & 70 & 68 & 69 & 84 & 83 & 85 \\
\hline 1991 & & 71 & 73 & 73 & 74 & 76 & 72 & 67 & 60 & 61 & 65 & 81 & 80 \\
\hline 1992 & & 70 & 71 & 67 & 70 & 71 & 60 & 56 & 55 & 60 & 66 & 79 & 79 \\
\hline 1993 & & 75 & 76 & 75 & 77 & 78 & 63 & 61 & 63 & 71 & 79 & 83 & 74 \\
\hline 1994 & & 71 & 75 & 78 & 76 & 76 & 67 & 61 & 56 & 58 & 70 & 79 & 73 \\
\hline 1995 & & 59 & 57 & 74 & 74 & 73 & 76 & 69 & 67 & 60 & 71 & 77 & 75 \\
\hline 1996 & & 72 & 73 & 78 & 72 & 72 & 71 & 64 & 65 & 64 & 75 & 76 & 73 \\
\hline 1997 & & 74 & 68 & 54 & 76 & 69 & 73 & 55 & 50 & 58 & 60 & 75 & 56 \\
\hline 1998 & & 73 & 73 & 76 & 78 & 77 & 72 & 67 & 64 & 53 & 60 & 71 & 73 \\
\hline 1999 & & 85 & 86 & 86 & 89 & 77 & 73 & 67 & 60 & 66 & 71 & 75 & 76 \\
\hline 2000 & & 68 & 69 & 84 & 80 & 73 & 73 & 68 & 66 & 78 & 72 & 78 & 83 \\
\hline 2001 & & 69 & 69 & 79 & 74 & 77 & 70 & 69 & 63 & 70 & 72 & 84 & 88 \\
\hline 2002 & & 72 & 74 & 75 & 82 & 80 & 78 & 69 & 66 & 66 & 72 & 77 & 82 \\
\hline 2003 & & 66 & 68 & 71 & 78 & 79 & 75 & 71 & 69 & 73 & 83 & 86 & 84 \\
\hline 2004 & & 82 & 79 & 75 & 85 & 84 & 75 & 79 & 67 & 67 & 77 & 80 & 82 \\
\hline 2005 & & 80 & 76 & 74 & 73 & 76 & 68 & 61 & 73 & 76 & 68 & 61 & 82 \\
\hline 2006 & & 72 & 71 & 79 & 77 & 73 & 70 & 65 & 59 & 61 & 75 & 78 & 76 \\
\hline 2007 & & 66 & 53 & 70 & 81 & 81 & 78 & 74 & 75 & 72 & 81 & 84 & 88 \\
\hline 2008 & & 84 & 81 & 83 & 86 & 82 & 79 & 75 & 78 & 80 & 88 & 93 & 90 \\
\hline 2009 & & 91 & 90 & 92 & 90 & 89 & 86 & 80 & 79 & 68 & 80 & 86 & 77 \\
\hline 2010 & & 69 & 72 & 78 & 85 & 87 & 86 & 87 & 78 & 82 & 85 & 92 & 91 \\
\hline 2011 & & 85 & 80 & 80 & 85 & 83 & 81 & 77 & 75 & 75 & 80 & 83 & 85 \\
\hline
\end{tabular}

\begin{tabular}{|lcccccccccccc|}
\hline PROMEDIO & 74,045 & 73,318 & 76,5 & 79,045 & 77,682 & 73,591 & 68,727 & 66,182 & 67,636 & 74,273 & 80,045 & 79,636 \\
VARIANZA & 55,407 & 64,126 & 54,159 & 30,225 & 25,035 & 38,696 & 58,38 & 59,24 & 58,231 & 57,835 & 44,589 & 57,231 \\
DESVIACIÓN & 7,4436 & 8,0079 & 7,3593 & 5,4977 & 5,0035 & 6,2206 & 7,6407 & 7,6967 & 7,631 & 7,6049 & 6,6775 & 7,5651 \\
IE & 0,9976 & 0,9878 & 1,0307 & 1,065 & 1,0466 & 0,9915 & 0,926 & 0,8917 & 0,9113 & 1,0007 & 1,0784 & 1,0729 \\
\hline
\end{tabular}

Fuente: elaboración propia 
Tabla 6. Base de datos variable humedad relativa, estación Mesitas

\begin{tabular}{|llllllll}
\hline & & & & ESTACIÓN : 2120646 MESITAS \\
Latitud & $0435 \mathrm{~N} X=\mathrm{N}=997800$ & Departamento & CUNDINAMARCA & Corriente & R. BOGOTA & Categoria & CP \\
Longitud & $7425 \mathrm{~W}$ Y=E=959500 & Municipio & EL COLEGIO & Cuenca & R. BOGOTÁ & Fecha Instalación & \#\#\#\#\#\#\# \\
Elevación & $1100 \mathrm{~m}$.s.n.m & Oficina Provincial & 13 TEQUENDAMA & & & Fecha Suspensión & \\
\hline
\end{tabular}

\begin{tabular}{|c|c|c|c|c|c|c|c|c|c|c|c|c|}
\hline AÑ̃O & ENERO & FEBRE & MARZO & ABRIL & MAYO & JUNIO & JULIO & AGOST & SEPTI & OCTUB & NOVIE & DICIE \\
\hline 1991 & 74 & 72 & 81 & 78 & 80 & 77 & 72 & 67 & 73 & 74 & 83 & 82 \\
\hline 1992 & 74 & 74 & 71 & 75 & 78 & 68 & 72 & 71 & 75 & 74 & 75 & 82 \\
\hline 1993 & 78 & 72 & 76 & 81 & 81 & 75 & 70 & 66 & 72 & 74 & 87 & 81 \\
\hline 1994 & 80 & 81 & 83 & 83 & 81 & 74 & 72 & 67 & 67 & 75 & 82 & 77 \\
\hline 1995 & 68 & 64 & 81 & 82 & 78 & 77 & 76 & 76 & 72 & 78 & 75 & 76 \\
\hline 1996 & 74 & 76 & 80 & 75 & 77 & 76 & 71 & 61 & 60 & 72 & 77 & 72 \\
\hline 1997 & 75 & 70 & 74 & 79 & 74 & 72 & 62 & 48 & 60 & 64 & 74 & 63 \\
\hline 1998 & 60 & 65 & 65 & 73 & 68 & 65 & 63 & 59 & 66 & 68 & 78 & 85 \\
\hline 1999 & 90 & 78 & 74 & 82 & 78 & 73 & 65 & 62 & 65 & 66 & 69 & 67 \\
\hline 2000 & 53 & 57 & 61 & 61 & 79 & 75 & 70 & 69 & 83 & 81 & 82 & 80 \\
\hline 2001 & 75 & 74 & 79 & 75 & 78 & 79 & 76 & 71 & 75 & 77 & 80 & 93 \\
\hline 2002 & 75 & 74 & 71 & 79 & 82 & 76 & 66 & 71 & 72 & 77 & 81 & 77 \\
\hline 2003 & 75 & 74 & 76 & 79 & 80 & 76 & 70 & 72 & 77 & 82 & 80 & 79 \\
\hline 2004 & 78 & 79 & 78 & 83 & 83 & 79 & 79 & 75 & 77 & 82 & 83 & 71 \\
\hline 2005 & 80 & 79 & 79 & 79 & 80 & 76 & 27 & 80 & 78 & 84 & 87 & 77 \\
\hline 2006 & 84 & 84 & 86 & 89 & 91 & 77 & 78 & 77 & 79 & 90 & 94 & 77 \\
\hline 2007 & 84 & 75 & 82 & 85 & 81 & 77 & 81 & 85 & 75 & 81 & 82 & 83 \\
\hline 2008 & 80 & 79 & 80 & 82 & 84 & 81 & 76 & 78 & 74 & 80 & 85 & 78 \\
\hline 2009 & 80 & 76 & 79 & 80 & 77 & 78 & 72 & 71 & 63 & 72 & 77 & 70 \\
\hline 2010 & 61 & 61 & 68 & 73 & 77 & 76 & 76 & 68 & 73 & 73 & 81 & 79 \\
\hline 2011 & 74 & 77 & 75 & 74 & 82 & 78 & 70 & 77 & 75 & 81 & 84 & 77 \\
\hline
\end{tabular}

\begin{tabular}{|lrrrrrrrrrrr|r|}
\hline PROMEDIO & 74,857 & 73,381 & 76,143 & 78,429 & 79,476 & 75,476 & 69,714 & 70,048 & 71,952 & 76,429 & 80,762 & 77,429 \\
VARIANZA & 69,646 & 43,474 & 36,503 & 31,578 & 17,868 & 12,63 & 115,63 & 64,045 & 36,998 & 37,483 & 27,8 & 40,15 \\
DESVIACIÓN & 8,3454 & 6,5935 & 6,0418 & 5,6195 & 4,2271 & 3,5539 & 10,753 & 8,0028 & 6,0826 & 6,1223 & 5,2726 & 6,3364 \\
IE & 0,9936 & 0,974 & 1,0106 & 1,041 & 1,0549 & 1,0018 & 0,9253 & 0,9297 & 0,955 & 1,0144 & 1,0719 & 1,0277 \\
\hline
\end{tabular}

Fuente: elaboración propia

Una vez se tienen las bases de datos completas, se procede a realizar el análisis de cada indicador. Es relevante recordar que debido a las condiciones del relieve, es posible establecer diferencias en cuanto a la ubicación de cada estación, tal como se muestra en la figura 6 .

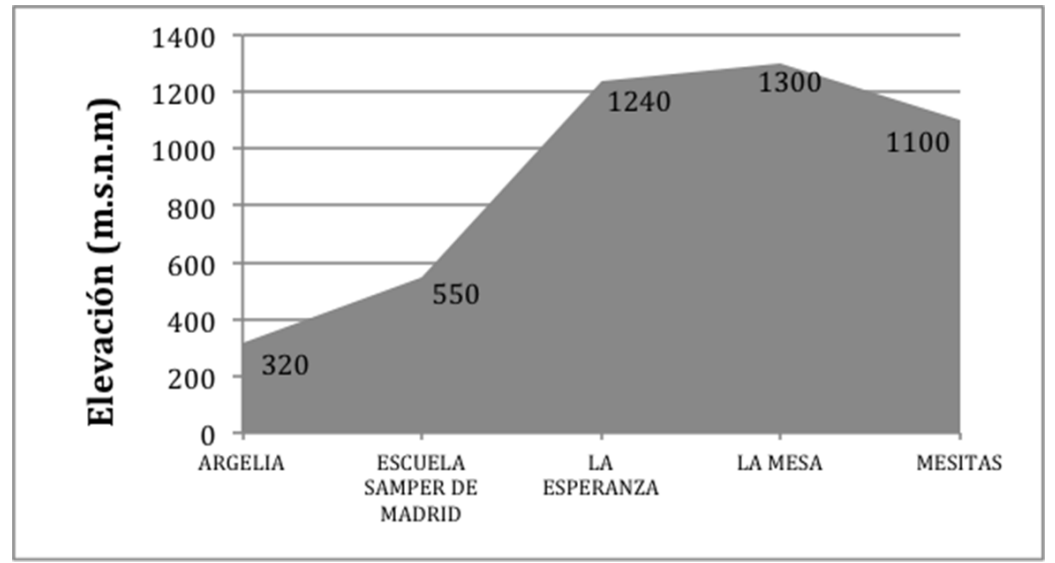

Figura 6. Elevación de las estaciones meteorológicas analizadas

Fuente: elaboración propia 
Esto permite plantear dos análisis de relación: el primero para las estaciones de Mesitas, La Mesa y La Esperanza, y el segundo para las estaciones de Escuela Samper y Argelia.

En forma general, al realizar un análisis de correlación entre cada una de las estaciones, frente a cada variable, es posible comparar y estudiar relaciones, más exactamente el grado de variación conjunta, estableciendo tendencias similares.

Tabla 7. Correlación de humedad relativa

\begin{tabular}{llllll}
\hline & Argelia & Samper & La Esperanza & La Mesa & Mesitas \\
\hline Argelia & 1 & & & & \\
\hline Samper & 0,26992401 & 1 & & & \\
\hline La Esperanza & 0,64545287 & 0,35103564 & 1 & & \\
\hline La Mesa & 0,52556497 & 0,41189951 & 0,6014204 & 1 & \\
\hline Mesitas & 0,25339014 & 0,23321026 & 0,39135609 & 0,50047296 & 1 \\
\hline
\end{tabular}

Fuente: elaboración propia

Este análisis aplicado a la variable humedad relativa debería mostrar una mayor correlación entre las estaciones ubicadas por encima de los $1000 \mathrm{~m}$ (La Esperanza, La Mesa y Mesitas), además por su cercanía. Sin embargo, solo se muestra una correlación baja entre la Mesa y La Esperanza (0,6014). Este hecho requiere un análisis minucioso del funcionamiento de las estaciones, equipos y estándares para la medición de estas variables.

Aplicando este mismo procedimiento, se analizó cada una de las bases de datos para cada variable en las diferentes estaciones relacionadas en este estudio.

\section{Conclusiones}

Evaluando las diferentes metodologías existentes, es posible establecer especificaciones de las estaciones en cuanto a medios tecnológicos, distribución y cantidad, lo cual permite realizar comparaciones entre estadígrafos con parámetros estadísticos confiables cuando hay similitud en los patrones (Campos, 1983).

Los datos evaluados de las estaciones del área de estudio muestran una gran variabilidad y baja correlación, de acuerdo con los resultados mostrados en la tabla 7. Esto implica una limitación enorme al momento de emplear metodologías para completar datos en cada una de variables meteorológicas consideradas en el presente estudio.

La evaluación de las bases de datos suministradas por la estaciones presenta diferentes fechas de inicio, rangos de valores y gran variación, lo que demuestra una gran dificultad para hacer esta evaluación, teniendo como referente las condiciones de la orografía del terreno, la altura sobre el nivel del mar y la varianza significativa en las condiciones del clima; por lo tanto, se debe considerar una área de estudio más amplia para minimizar la incertidumbre del comportamiento de las variables.

De acuerdo con lo manifestado por Martín, López y Calera (2005), luego de una experiencia adquirida por muchos años, el buen funcionamiento de una estación requiere el control y el análisis de datos que se proporciona en tiempo real, para lograr detectar anomalías en su funcionamiento y realizar los correctivos correspondientes (Martin, Lopez \& Calera, 2005, p. 257). 


\section{Referencias}

Alarcón, J. C., Arango, G. J., Benavídez, H., Cano, C., Leyva, P., Ojeda, D., Pérez, S., Ponce, E., Jaramillo, O., Cárdenas, I., Rozo, E., Suárez, O. \& Cuevas, H. (2001). Primera comunicación nacional ante la Convención Marco de las Naciones Unidas sobre el Cambio Climático. CG-1687. Colombia

Bárcena Hinojal, I. et ál. (2009). Energía y deuda ecológica: transnacionales, cambio climático y alternativas. Barcelona: Icaria. Benítez G. F. (1998). Inventario de gases de efecto invernadero, fuentes y sumideros, año 1990 Bogotá

Benítez G. F. (2000). Opciones para la reducción de emisiones de gases de efecto invernadero en Colombia. Bogotá: Grupo Intergubernamental de Expertos sobre el Cambio Climático.

Cabrera, R. (s. f.). Lecciones teóricas de biofísica. Universidad de Buenos Aires. Recuperado el 22 de julio del 2013, de http://neuro.qi.fcen.uba.ar/ricuti/No_me_salen/FLUIDOS/FT_humedad.html.

Corporación Autónoma Regional de Cundinamarca (CAR) (2000). Contribución a la mitigación del cambio climático y al desarrollo forestal de la región del páramo de Guerrero por parte de la CAR. CAR-0870. Imprenta Nacional, Bogota.

Corporación Autónoma Regional de Cundinamarca (CAR) (2009). Cambio climático MDL Carbono neutral. CAR-1086. Imprenta Nacional, Bogota.

Campos, D. (1983). Procesos del ciclo hidrológico. San Luis Potosi, México: Universia Potosina.

Costa Posada, C. (2008). La adaptación al cambio climático en Colombia. Revista Virtual Redesma, 2 (octubre).

De Alba, E. (2004). La Convención Marco de las Naciones Unidas sobre Cambio Climático. En J. Martínez y A. Fernández Bremauntz (Comps.), Cambio climático: una visión desde México. México: Instituto Nacional de Ecología y Secretaría de Medio Ambiente y Recursos Naturales.

Ferrán, B. \& Julio, D. (2006). Cambio climático y salud pública: escenarios después. Valencia: Escuela Valenciana de Estudios para la Salud.

Guarín, N. (2002). Estadística aplicada. Recuperado el 20 de octubre del 2012, de http://fcbi.unillanos.edu.co/ proyectos/Facultad/php/tutoriales/upload_tutos/Curso\% 20De\% 20Estadistica\% 20Aplicada.pdf

Hanke, J. \& Reitsch, A. (1996). Pronósticos en los negocios. México: Pearson Educación.

Infante, S., Ortega, J. \& Cedeño, F. (2008). Estimacion de datos faltantes en estaciones metereológicas de Venezuela vía un modelo de redes neuronales. Revista Climatologica, 8, 51-70.

Instituto de Hidrología, Meteorología y Estudios Ambiental (Ideam) (2007). Memorias de la Primera Conferencia Internacional de Cambio Climático: Impacto en los Sistemas de Alta Montaña. CG-2481. Bogotá: Ideam.

Instituto de Hidrología, Meteorología y Estudios Ambiental (Ideam) (2008a). Alertas tempranas: una estrategia para reducir impactos de los desastres naturales y prepararnos para el cambio climático CG-3168. Bogotá: Ideam.

Instituto de Hidrología, Meteorología y Estudios Ambiental (Ideam) (2008b). Escenarios de cambio climático para 24 regiones de Colombia. Recuperado el 20 de octubre del 2012, de http://www.cambioclimatico.gov.co/ documentos/INFORME\% 20FINAL

Instituto de Hidrología, Meteorología y Estudios Ambiental (Ideam) (2010). Estrategia nacional de educación, formación y sensibilización de públicos sobre cambio climático. Bogotá: Ideam.

Instituto Internacional de Investigación sobre Políticas Alimentarias (IFPri) (2009). Cambio climático. El impacto en la agricultura y los costos de adaptación. Washington, D. C: IFPRI.Intergovernmental Panel on Climate Change (IPCC) (2007). Informe del Grupo Intergubernamental de Expertos sobre el Cambio Climático 2007. Ginebra, Suiza: PNUMA. 
Martin, F., López, P, \& Calera, A. (2005). Agua y agronomía. Madrid: Mundi-Prensa.

Nieves, A. \& Dominig, F. (2010). Probabilidad y estadistica para ingeniería. Mexico: McGraw-Hill.

Rey, J. C. (2007). Estudio comparativo de diferentes métodos de interpolación ara la realización de mapas de precipitación en la isla de La Gomera. Geogaceta, (42), 131-134.

Spiegel, M., Schiller, J. \& Srinivasan, A. (2010). Probabilidad y estadística. Mexico: McGraw-Hill.Campos, D. (1983). Procesos del ciclo hidrologico. San Luis Potosi: Universia potosina.

GUARIN, N. (15 de 09 de 2002). Estadistica Aplicada. Recuperado el 20 de 10 de 2012, de http://fcbi.unillanos. edu.co/proyectos/Facultad/php/tutoriales/upload_tutos/Curso\%20De\%20Estadistica\%20Aplicada.pdf

IDEAM. (29 de 09 de 2008). Escenarios de cambio climático para 24 regiones de Colombia. Recuperado el 20 de 10 de 2012, de http://www.cambioclimatico.gov.co/documentos/INFORME\%20FINAL

IFPRI. (2009). Cambio Climatico. El impacto en la agricultura y los costos de adaptación. Washington, D.C: IFPRI.

Infante, S., Ortega, J., \& Cedeño, F. (2008). Estimacion de datos faltantes en estaciones metereológicas de Venezuela vía un modelo de redes neuronales. Revista Climatologica Vol 8 , 51-70.

IPCC. (2007). Informe del Grupo Intergubernamental de Expertos sobre el Cambio Climático (PNUMA Y OMM) 2007. GINEBRA; SUIZA: PNUMA.

Hanke, J., \& Reitsch, A. (1996). Pronosticos en los negocios. México: Pearson Educación.

Nieves, A., \& Dominig, F. (2010). Probabilidad y Estadistica para Ingenieria. Mexico: Mc Graw Hill.

Martin, F., Lopez, P., \& Calera, A. (2005). Agua y Agronomía. España: Mundi-Premsa.

Spiegel, M., Schiller, J., \& Srinivasan, A. (2010). Probabilidad y Estadistica. Mexico: Mc Graw Hill.

Rey, J. C. (2007). Estudio comparativo de diferentes métodos de interpolación ara la realización de mapas de precipitación en la isla de La Gomera. GEOGACETA, 131-134. 
